\title{
Effects of 16-Form Wheelchair Tai Chi on the Autonomic Nervous System among Patients with Spinal Cord Injury
}

\author{
Yan Qi $\mathbb{D}^{1,2}$ Haixia Xie $\mathbb{D D}^{1}{ }^{1}$ Yunlin Shang $\left(\mathbb{D},{ }^{1}\right.$ Lejun Wang $\mathbb{D}^{3},{ }^{3}$ Ce Wang $\mathbb{D}^{\circ},{ }^{3}$ Yalin He, ${ }^{1}$ \\ Wenxin Niu $\mathbb{D}^{2},{ }^{2}$ and Dongliang Shi $\mathbb{I D}^{1}$ \\ ${ }^{1}$ Yangzhi Rehabilitation Hospital, Tongji University School of Medicine, Shanghai 201619, China \\ ${ }^{2}$ Department of Rehabilitation Therapy, Tongji University School of Medicine, Shanghai 200092, China \\ ${ }^{3}$ Department of Physical Education, Tongji University, Shanghai 200092, China
}

Correspondence should be addressed to Wenxin Niu; niu@tongji.edu.cn and Dongliang Shi; shi_dongliang@126.com

Received 15 October 2020; Revised 4 November 2020; Accepted 17 November 2020; Published 28 November 2020

Academic Editor: Xue-Qiang Wang

Copyright ( 92020 Yan Qi et al. This is an open access article distributed under the Creative Commons Attribution License, which permits unrestricted use, distribution, and reproduction in any medium, provided the original work is properly cited.

Objective. This study aims to investigate the effects of 16-form Wheelchair Tai Chi (WCTC16) on the autonomic nervous system among patients with spinal cord injury (SCI). Methods. Twenty patients with chronic complete thoracic SCI were recruited. Equivital life monitoring system was used to record and analyze heart rate variability (HRV) of patients for five minutes before and after five consecutive sets of WCTC16, respectively. The analysis of HRV in the time domain included RR intervals, the standard deviation of all normal RR intervals (SDNN), and the root mean square of the differences between adjacent NN intervals (RMSSD). The analysis of HRV in the frequency domain included total power (TP), which could be divided into very-lowfrequency area (VLFP), low-frequency area (LFP), and high-frequency area (HFP). The LF/HF ratio as well as the normalized units of LFP (LFPnu) and HFP (HFPnu) reflected the sympathovagal balance. Results. There was no significant difference in RR interval, SDNN, RMSSD, TP, HEP, VLFP, and LFP of SCI patients before and after WCTC16 exercise $(P>0.05)$. LFPnu and HF peak decreased, while HFPnu and LF/HF increased in SCI patients after WCTC16 exercise. The differences were statistically significant $(P<0.001)$. Conclusion. WCTC16 can enhance vagal activity and decrease sympathetic activity so that patients with chronic complete thoracic SCI can achieve the balanced sympathovagal tone.

\section{Introduction}

Spinal cord injury (SCI) refers to damage to the structure and function of the spinal cord due to various reasons, resulting in sensory, motor, and autonomic dysfunction below the injury level [1]. Autonomic dysfunction after SCI is a common complication with an incidence of $43 \%$ [2], which can lead to stroke, convulsions, cardiac arrest, and death [3].

Autonomic dysfunction is a potentially life-threatening complication that occurs in patients with SCI [4]. Autonomic disorders are currently the main cause of death in patients with chronic SCI [5]. Some scholars have pointed out that autonomic dysfunction can reach a relatively "internally stable" state through complex adjustments in patients with chronic SCI [6-8]. For example, after the sympathetic innervation of the heart in patients with chronic quadriplegia is interrupted, the brain can also send signals that inhibit the activity of the parasympathetic nerve in order to maintain the homeostasis of the sympathetic-vagus nerve and simultaneously provoke reduction of sympathetic and parasympathetic nerve activity meaning autonomic reflex disorders [7].

Exercise is particularly important for the patients with SCI for that it can promote the recovery of neurological function and functional compensation, improve motor function and activities of daily living, enhance cardiovascular and respiratory functions, and develop balance function [9-13]. However, inappropriate exercise can induce autonomic disorders. It has been reported that about $90 \%$ of tetraplegic athletes induce abnormal autonomic reflexes in order to increase performance [14]. 
Tai Chi is a treasure of Chinese culture and has been widely applied in rehabilitation $[15,16]$. A large number of studies have confirmed that traditional Tai Chi exercises can improve the cardiopulmonary function of practitioners $[17,18]$, but the mechanism of autonomic nervous system regulation that improves cardiovascular function is still unclear, and the results were often controversial $[19,20]$.

Because traditional Tai Chi is performed in a standing posture, many practitioners have difficulty in completing it due to various reasons. Therefore, based on the essence of traditional Tai Chi, wheelchair Tai Chi was proposed. It retains the movements of the upper limbs and torso and reduces or even does not have the movements of the lower limbs. It is suitable for a wider range of people, especially for the patients with SCI [21]. Similarly, it is unclear whether wheelchair Tai Chi can enhance the balance of autonomic nerve activity in chronic SCI.

In terms of autonomic nerve function assessment, heart rate variability (HRV), the 24-hour sinus heart rate having a certain degree of change over time, is getting more and more attention. HRV reflects the activity of the autonomic nervous system and quantitatively evaluates the tension and balance of the cardiac sympathetic nerve and vagus nerve, thereby revealing the regulation or influence of the autonomic nervous system on the cardiovascular system. HRV is a reliable, sensitive, and repeatable noninvasive detection method in SCI $[22,23]$. Its time domain and frequency domain analysis method are basically mature in theory, and its clinical application range is wide. The frequency domain method can quantitatively indicate the modulation of the heart by the sympathetic nerve and the vagus nerve.

This study investigated the HRV indicators to explore the effect of 16-form Wheelchair Tai Chi (WCTC16) on the autonomic nervous system of patients with SCI in order to provide theoretical basis for safe and effective scientific fitness.

\section{Methods}

2.1. Participant Recruitment. The immediate effect of WCTC16 on autonomic nervous modulation in patients with chronic SCI was investigated. Twenty patients with complete thoracic SCI were recruited. These participants were right-handed SCI inpatients recruited during their recovery period from a rehabilitation hospital. The inclusion criteria were people who met the diagnostic criteria for complete SCI according to the American Spinal Injury Association [24]. They were all between 20 and 60 years old, able to communicate and follow instructions, and able to maintain a sitting posture for more than 30 minutes in a wheelchair.

The exclusion criteria were people with an unstable spine, metastatic cancer, spine tumor, serious cardiopulmonary disease, poorly controlled hypertension, poorly controlled trunk and upper limb hypertonia, or serious complications related to SCI, such as pressure ulcers and contracture.
The protocol was approved by the ethics committee of the rehabilitation, hospital and written informed consent was obtained from all participants before study. This study was registered on the Chinese Clinical Trial Registry (ChiCTR-1900023734).

2.2. Test Equipment. The Equivital series of dynamic vital signs monitors (Equivital Life Monitor HIDA3330-131P16, UK) monitored the participants' real-time heart rate, respiratory rate, body position, and body temperature; analyzed the HRV RR interval and the standard deviation of normal RR interval (SDNN), total power (TP), lowfrequency power (LFP), high-frequency power (HFP), standardized $\mathrm{HF}$, and other indicators of the changes using the built-in software; and then inferred the regulatory mechanism of the autonomic nervous system (sympathetic nerve and vagus nerve). The reliability and validity of the instrument had been confirmed [23].

2.3. Testing Method. Participants should avoid strenuous exercise within 24 hours before the test and smoking or drinking during the 2 hours before the test. They should have proper rest about 30 minutes and no full meals. Loose clothing was suitable for exercise during each test. After the participants arrived at the test site, they sat quietly to restore their physical functions. When they were sitting quietly, they were instructed to breathe calmly and steadily, stay in the surrounding environment away from noise interference, forbid talking with the participants, and keep the posture as relaxed and stable as possible.

Then, they wore a dynamic vital sign monitor, and HRV indicators were collected in a resting state for 5 minutes, as shown in Figure 1. The participants completed the warm-up with music, five consecutive WCTC16 exercises, and finishing exercises. After the recovery period, HRV indicators were collected for 5 minutes. After the test, all the data were imported into the automatic analysis software to explore the regulation mechanism of autonomic nerve function during the resting or pre-WCTC16 period before exercise and during the recovery or post-WCTC16 period after exercise. The rhythm and duration of WCTC16 exercises were controlled by 5-minute Tai Chi music with action prompts.

\subsection{Test Parameters}

\subsubsection{Time Domain Index of $H R V$}

$\mathrm{RR}$ interval: the average value of the normal RR interval, in $\mathrm{ms}$

SDNN: the standard deviation of the normal RR interval, in $\mathrm{ms}$

RMSSD: the root mean square of the difference between adjacent RR intervals basically reflecting the average absolute value of the RR interval changes per 


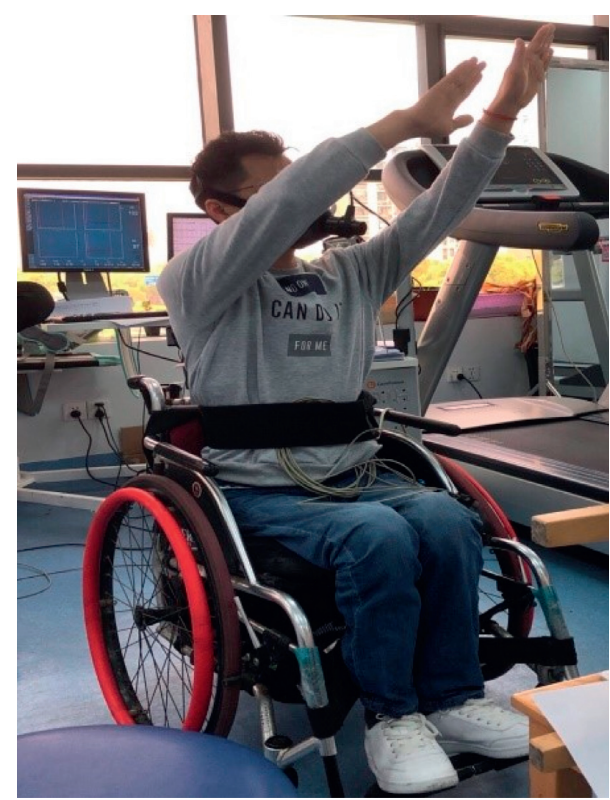

FIGURE 1: Dynamic vital sign monitor during 16-form Wheelchair Tai Chi.

stroke was sensitive to irregular heartbeats and/or heartbeat waveform marking errors, in ms

\subsubsection{Frequency Domain Index of $H R V$}

Total power (TP): it is the total variance of $\mathrm{HRV}$, in $\mathrm{ms}^{2}$ Very-low-frequency power (VLFP): it is the amplitude of the basic heart rate oscillation of the heart rate mode from once every 25 seconds to once every 5 minutes $(0.003-0.04 \mathrm{~Hz})$, in $\mathrm{ms}^{2}$

Low-frequency power (LFP): it is the amplitude of the heart rate oscillation in the range of 3-9 cycles per minute $(0.04-0.15 \mathrm{~Hz})$, in $\mathrm{ms}^{2}$

High-frequency power (HFP): it is the amplitude of the heart rate oscillation in the range of 9-24 cycles per minute $(0.15-0.40 \mathrm{~Hz}$, which is the typical adult breathing frequency range), in $\mathrm{ms}^{2}$

$\mathrm{LF} / \mathrm{HF}$ ratio: it is a ratio, usually referred to as "sympathetic-vagus" balance

Normalization unit of low-frequency power (LFPnu): it is the ratio of LFP to HRV

Normalization unit of high-frequency power (HFPnu): it is the ratio of HFP to HRV

2.5. Statistical Analyses. Each parameter was represented by the mean \pm standard deviation (SD), and the data met the homogeneity of variance test and the normality test, and the paired sample $t$-test before and after itself was used. $\alpha=0.05$ was set, and $P<0.05$ indicated that the difference was statistically significant using IBM-SPSS 23.0 software (SPSS Inc., Chicago, IL, USA) for statistical analyses.

\section{Results}

3.1. Time Domain Analysis. All the analysis results are listed in Table 1. Though WCTC16 exercise increased RR interval, SDNN, and RMSSD of SCI patients, all these changes were not statistically significant $(P>0.05)$.

3.2. Frequency Domain Analysis. As shown in Table 1, WCTC exercise increased TP and HFP, while decreased VLFP and LFP of SCI patients, but all these changes were not statistically significant $(P>0.05)$.

Compared with that before exercise, LFPnu and HFP peak decreased, while HFPnu increased along with LF/HF in SCI patients after WCTC16 exercise, and all these differences were statistically significant $(P<0.001)$.

\section{Discussion}

The new evidence-based exercise guidelines for SCI adults updated by the International Spinal Cord Association put more emphasis on guidelines for cardiovascular disease in SCI patients [25]. It was recommended to do aerobic exercise of medium-to-high intensity at least 2 times a week for more than 20 minutes each time and 2 times a week for each major functional muscle group, 3 groups each time mediumto-high intensity strength training (strongly recommended), to improve cardiorespiratory fitness and muscle strength. It was recommended to do aerobic exercise of medium-to-high intensity at least 3 times a week for more than 30 minutes each time (recommendation with conditions) to improve cardiovascular metabolism.

The human cardiovascular system is mainly dominated by sympathetic and parasympathetic nerves. Xiong et al. [19] found that Tai Chi could improve the HRV of middle-aged and elderly people and optimize the heart rate status. On the basis of cardiac rehabilitation, combined with Tai Chi, the sensitivity of vagus nerve reflex in patients with coronary atherosclerotic heart disease was improved, but HRV had no significant change [20]. Chen et al. [20] concluded that Tai Chi could enrich the quality of life of patients with coronary heart disease, but it had no effect on HRV.

The rMSSD is an indicator that reflects the tone of the vagus nerve [21]. In this study, the rMSSD increased slightly after WCTC16, but it was not statistically significant. As an indicator reflecting the tension of sympathetic and vagus nerve, SDNN is often used to evaluate the overall degree of autonomic nervous system damage and recovery [21]. SDNN and RR interval increased slightly following WCTC16, and they were still not statistically significant. The results of this study are consistent with the results of many authors [26-28]. The reason might be that the above indicators were not sensitive to short-term Wheelchair Tai Chi [29].

The changes of TP, VLFP, LFP, and HFP after WCTC16 exercise were not statistically significant. However, after WCTC16 exercise, the LF/HF ratio, LFPnu, and peak of HF decreased, and HFPnu increased, all of which were 
TABLE 1: HRV between pre- and post-WCTC16 (mean \pm SD).

\begin{tabular}{lcccc}
\hline $\begin{array}{l}\text { Parameter } \\
\text { (unit) }\end{array}$ & Pre-WCTC16 & Post-WCTC16 & $t$ & $P$ \\
\hline $\begin{array}{l}\text { RR interval } \\
(\mathrm{ms})\end{array}$ & $604.89 \pm 47.56$ & $607.33 \pm 58.12$ & -0.445 & 0.662 \\
SDNN (ms) & $34.96 \pm 11.89$ & $35.29 \pm 17.76$ & -0.183 & 0.857 \\
RMSSD (ms) & $39.87 \pm 19.41$ & $41.37 \pm 25.07$ & -0.936 & 0.363 \\
TP $\left(\mathrm{ms}^{2}\right)$ & $749.00 \pm 536.62$ & $754.56 \pm 586.90$ & -0.369 & 0.717 \\
VLFP $\left(\mathrm{ms}^{2}\right)$ & $51.94 \pm 21.92$ & $50.72 \pm 25.89$ & 0.813 & 0.427 \\
LFP $\left(\mathrm{ms}^{2}\right)$ & $340.06 \pm 234.79$ & $337.28 \pm 258.66$ & 0.392 & 0.700 \\
HFP $\left(\mathrm{ms}^{2}\right)$ & $339.50 \pm 236.88$ & $372.28 \pm 313.18$ & -1.455 & 0.164 \\
LFPnu & $61.75 \pm 11.21$ & $52.89 \pm 17.48$ & 12.753 & $<0.001$ \\
HFPnu & $38.25 \pm 11.21$ & $46.81 \pm 17.48$ & -10.835 & $<0.001$ \\
LF/HF & $2.24 \pm 0.68$ & $1.49 \pm 0.85$ & 7.565 & $<0.001$ \\
HF peak & $0.29 \pm 0.08$ & $0.23 \pm 0.08$ & 7.526 & $<0.001$ \\
$(\mathrm{~Hz})$ & & & & \\
\hline
\end{tabular}

HRV, heart rate variability; WCTC16, 16-form Wheelchair Tai Chi; TP, total power; VLFP, power in very-low-frequency area; LFP, power in lowfrequency area; HFP, power in high-frequency area; LFPnu, normalization unit of low-frequency power; HFPnu, normalization unit of high-frequency power; LF/HF, low-frequency area divided by high-frequency area; HF peak, peak of high-frequency area.

statistically significant. These results were consistent with the results of Buker [30].

As an objective HRV indicator, LF/HF ratio is usually used to reflect the overall sympathetic and vagal balance state [21]. In this study, the LF/HF ratio dropped from 2.24 in the rest or pre-WCTC16 period to 1.49 in the recovery or post-WCTC16 period, which was closer to the ratio of healthy adults [31]. It suggested that WCTC16 helped to restore the sympathetic-vagal balance to the level of healthy adults. Giagkoudaki et al. [32] also found that the excitability of the vagus nerve in patients with Down syndrome increased after aerobic training, the LF/HF ratio decreased (2.45 1.72), and the balance of sympathetic-vagus nerve tension revived. Some authors showed that decreased cardiac vagus nerve activity might lead to increased mortality in patients with myocardial infarction [33]. In this study, WCTC16 increased the parasympathetic nerve activity of SCI patients. It was speculated that WCTC16 could improve autonomic nerve control and reduce mortality in SCI patients.

HFP reflects the vagus nerve regulation function [34]. In this study, HFPnu increased after WCTC16 exercise. It showed that the activity of the vagus nerve increased. LFP reflects the comprehensive regulation results of parasympathetic and sympathetic efferent nerve activity and is affected by baroreflex activity [35]. In this study, significantly reduced LFPnu suggested common regulation of vagussympathetic nerves and the balance function between them.

Tai Chi exercise emphasizes the training of "Qi" (bioenergy) by breathing and the mind in body. The peak of HF can reflect the influence of exercise on breathing rate [36]. The Wheelchair Tai Chi exercise also requires deep and slow breathing. In this study, the peak of HF of subjects after WCTC16 decreased from 0.29 before exercise to 0.23 during the recovery period, implying that the activity of the vagus nerve relatively increased $[28,37]$.
The decrease in the ratio of LFPnu and LF/HF was the result of the combined effect of decreased sympathetic nerve activity and increased parasympathetic nerve activity. It was related to the intrinsic vagus nerve reactivity, baroreceptor sensitivity, and the adaptive state accompanying spinal cord injury in patients with SCI, or a combination of the above. In addition to the abovementioned regulation of the autonomic nervous system, other factors have to be considered [38]. For example, motor stimulation interrupts the central command from the motor cortex of the brain; the stimulation of mechanoreceptors, baroreceptors, and thermoreceptors is reduced; hormone levels are disordered [39]. These are consistent with the results of related research on the effect of standing Tai Chi on heart disease in the healthy elderly [40].

WCTC16 is a moderate-to low intensity exercise for patients with complete thoracic SCI. During exercise, SCI patients have increased heart rate, blood pressure, and faster breathing, which are manifested as a relative increase in dominant sympathetic nerve activity with decreasing vagus nerve activity. After WCTC16 exercise, the parasympathetic nerve activity significantly increased, the sympathetic nerve activity decreased, and the sympathetic-vagus nerve balance improved. It showed that the body's autonomic nervous "automatic regulation" was on and a process of establishing a new balance. It is important for SCI patients with autonomic dysfunction, especially for patients whose sympathetic and parasympathetic nerve activity was reduced at the same time, to restore the dynamic balance of autonomic nervous function.

During WCTC16 exercise, it is necessary to coordinate breathing and exercise under the guidance of mind. This is consistent with the principles of cardiopulmonary rehabilitation for SCI patients. Although the primary center of the visceral reflex activity was damaged, exercise could stimulate the lower brainstem, hypothalamus, and cerebral cortex, and the combined effects developed the function of autonomic nerves and the role of system regulation. Therefore, WCTC16 had a positive role on improving the cardiopulmonary function, preventing, and treating SCI complications.

However, this study has several limitations. Firstly, the relationship between LF power of HRV and cardiac autonomic function has still been unclear. LF power seems to provide an index not of cardiac sympathetic tone but of baroreflex function [41]. This would limit the interpretation of LF and LF/HF ratio to some extent [42]. Secondly, blood pressure has some effect on HRV, but blood pressure has not been investigated in this study. Thus, it is difficult to explore the relationship between the autonomic control and blood pressure regulation [43]. So, it is incomplete to make a thorough inquiry about the mechanism of WCTC16 affecting HRV. In future, we shall design and perform correlational research studies to solve these problems.

\section{Conclusion}

The WCTC16 might enhance vagal activity and decrease sympathetic activity with balanced sympathovagal tone in 
patients with complete thoracic SCI. By recovering the autonomic dysfunction and hyper-reflexia causing lifethreatening complication during exercise or postural changes, SCI patients could achieve a relatively "internally stable" state through complex regulation. In addition to the increase in the sympathetic nerve activity of patients during WCTC16, the parasympathetic nerve activity was significantly increased and dominant through the recovery period. In a word, WCTC16 exercise might enable the body to activate the automatic regulation of the autonomic nervous system and present a new dynamic balance.

$\begin{array}{ll}\text { Abbreviations } \\ \text { SCI: } & \text { Spinal cord injury } \\ \text { WCTC16: } & \text { 16-form Wheelchair Tai Chi } \\ \text { HRV: } & \text { Heart rate variability } \\ \text { SDNN: } & \text { The standard deviation of the normal RR interval } \\ \text { rMSSD: } & \text { The root mean square of the difference between } \\ & \text { adjacent RR intervals } \\ \text { TP: } & \text { Total power } \\ \text { VLFP: } & \text { Very-low-frequency power } \\ \text { LFP: } & \text { Low-frequency power } \\ \text { HFP: } & \text { High-frequency power } \\ \text { LFPnu: } & \text { Normalization unit of low-frequency power } \\ \text { HFPnu: } & \text { Normalization unit of high-frequency power. }\end{array}$

\section{Data Availability}

The data used to support the findings of this study are available from the corresponding author upon request.

\section{Ethical Approval}

The study protocol was in accordance with the Declaration of Helsinki and was approved by the Yang Zhi Rehabilitation Hospital Affiliated to Tongji University Ethics Committee (no. YZ2016001), and the authors registered the study on the Chinese Clinical Trial Registry (no. ChiCTR-1900023734).

\section{Conflicts of Interest}

The authors declare that they have no conflicts of interest regarding the publication of this paper.

\section{Authors' Contributions}

Yan Qi and Haixia Xie contributed equally to this work.

\section{Acknowledgments}

This study was supported by the Songjiang District Science and Technology Research Project (no. 19SJKJGG11), Shanghai Traditional Chinese Medicine Inheritance and Technological Innovation Project (no. ZYKC2019032), and National Natural Science Foundation of China (no. 81772432). The authors thank all the participants for agreeing to join this study. The authors thank all physicians, instructors, patient advisers, and other contributors.

\section{References}

[1] A. E. Ropper and A. H. Ropper, "Acute spinal cord compression," New England Journal of Medicine, vol. 376, no. 14, pp. 1358-1369, 2017.

[2] M. D. Stillman, J. Barber, S. Burns, S. Williams, and J. M. Hoffman, "Complications of spinal cord injury over the first year after discharge from inpatient rehabilitation," Archives of Physical Medicine and Rehabilitation, vol. 98, no. 9, pp. 1800-1805, 2017.

[3] H. Cowan, "Autonomic dysreflexia in spinal cord injury," Nursing Times, vol. 111, no. 44, pp. 22-24, 2015.

[4] E. Partida, E. Mironets, S. Hou, and V. J. Tom, "Cardiovascular dysfunction following spinal cord injury," Neural Regeneration Research, vol. 11, no. 2, pp. 189-194, 2016.

[5] A. A. Phillips and A. V. Krassioukov, "Contemporary cardiovascular concerns after spinal cord injury: mechanisms, maladaptations, and management," Journal of Neurotrauma, vol. 32, no. 24, pp. 1927-1942, 2015.

[6] B. Guadalupe, G. S. Gabriel, A. Ibarra et al., "Cardiovascular alteration after spinal cord injury: an overview," Current Medicinal Chemistry, vol. 2, pp. 133-148, 2004.

[7] Y.-H. Wang, T.-S. Huang, J.-L. Lin et al., "Decreased autonomic nervous system activity as assessed by heart rate variability in patients with chronic tetraplegia," Archives of Physical Medicine and Rehabilitation, vol. 81, no. 9, pp. 1181-1184, 2000.

[8] F. A. A. Gondim, A. C. A. Lopes Jr., G. Oliveira et al., "Cardiovascular control after spinal cord injury," Current Vascular Pharmacology, vol. 2, no. 1, pp. 71-79, 2004.

[9] H. Huang, W. Young, S. Skaper et al., "Clinical neurorestorative therapeutic guidelines for spinal cord injury (IANR/CANR version 2019)," Journal of Orthopaedic Translation, vol. 20, pp. 14-24, 2020.

[10] L. D. Hachem, C. S. Ahuja, and M. G. Fehlings, "Assessment and management of acute spinal cord injury: from point of injury to rehabilitation," The Journal of Spinal Cord Medicine, vol. 40, no. 6, pp. 665-675, 2017.

[11] C. R. West, M. A. Crawford, M.-S. Poormasjedi-Meibod et al., "Passive hind-limb cycling improves cardiac function and reduces cardiovascular disease risk in experimental spinal cord injury," The Journal of Physiology, vol. 592, no. 8, pp. 1771-1783, 2014.

[12] C. L. Rochester, I. Vogiatzis, A. E. Holland et al., “An official American thoracic society/European respiratory society policy statement: enhancing implementation, use, and delivery of pulmonary rehabilitation," American Journal of Respiratory and Critical Care Medicine, vol. 192, no. 11, pp. 1373-1386, 2015.

[13] J. B. Chen, Z. X. Jin, J. J. Yao et al., "Influence of the intelligent standing mobile robot on lower extremity physiology of complete spinal cord injury patients," Medicine in Novel Technology and Devices, vol. 7, Article ID 10045, 2020.

[14] A. Krassioukov, "Autonomic dysreflexia: current evidence related to unstable arterial blood pressure control among athletes with spinal cord injury," Clinical Journal of Sport Medicine: Official Journal of the Canadian Academy of Sport Medicine, vol. 22, no. 1, pp. 39-45, 2012.

[15] J. W. Duan, K. Wang, T. B. Chang, L. Wang, S. Zhang, and W. Niu, "Tai Chi is safe and effective for the hip joint: a biomechanical perspective," Journal of Aging and Physical Activity, vol. 28, no. 3, pp. 415-425, 2020.

[16] X. Q. Wang, Y. L. Pi, P. J. Chen et al., "Traditional Chinese exercise for cardiovascular diseases: systematic review and 
meta-analysis of randomized controlled trials," Journal of the American Heart Association, vol. 5, no. 3, Article ID e002562, 2016.

[17] Q. Gu, S.-J. Wu, Y. Zheng et al., "Tai Chi exercise for patients with chronic heart failure: a meta-analysis of randomized controlled trials," American Journal of Physical Medicine \& Rehabilitation, vol. 96, no. 10, pp. 706-716, 2017.

[18] L. A. Lipsitz, E. A. Macklin, T. G. Travison et al., "A cluster randomized trial of Tai Chi vs health education in subsidized housing: the MI-WiSH study," Journal of the American Geriatrics Society, vol. 67, no. 9, pp. 1812-1819, 2019.

[19] X. L. Xiong, C. Y. Mu, and Y. N. Feng, "The influence of Taijiquan on the depression and heart rate variability of middle-aged and old people," China Medical Devices, vol. 32, pp. 148-149, 2017.

[20] Z. Chen, H. D. Yu, and N. N. Zhang, "Effect of Tai Chi exercise on the physiological indexes and quality of life of patients with coronary heart disease: a systematic review," Nursing Journal of Chinese People's Liberation Army, vol. 35, no. 1, pp. 8-14, 2018.

[21] Y. Qi, X. Zhang, Y. C. Zhao et al., "The effect of wheelchair Tai Chi on balance control and quality of life among survivors of spinal cord injuries: a randomized controlled trial," Complementary Therapies in Clinical Practice, vol. 33, pp. 7-11, 2018.

[22] H. M. Melo, T. C. Martins, L. M. Nascimento et al., "Ultrashort heart rate variability recording reliability: the effect of controlled paced breathing," Annals of Noninvasive Electrocardiology, vol. 23, no. 5, Article ID e12565, 2018.

[23] Y. Liu, S. H. Zhu, G. H. Wang, F. Ye, and P. Z. Li, "Validity and reliability of multiparameter physiological measurements recorded by the equivital lifemonitor during activities of various intensities," Journal of Occupational and Environmental Hygiene, vol. 10, no. 2, pp. 78-85, 2013.

[24] C. Schuld, S. Franz, K. Brüggemann et al., "International standards for neurological classification of spinal cord injury: impact of the revised worksheet (revision 02/13) on classification performance," The Journal of Spinal Cord Medicine, vol. 39, no. 5, pp. 504-512, 2016.

[25] K. A. Martin Ginis, J. W. van der Scheer, A. E. LatimerCheung et al., "Evidence-based scientific exercise guidelines for adults with spinal cord injury: an update and a new guideline," Spinal Cord, vol. 56, no. 4, pp. 308-321, 2018.

[26] Y. K. Jan, M. Anderson, J. Soltani, S. Burns, and R. D. Foreman, "Comparison of changes in heart rate variability and sacral skin perfusion in response to postural changes in people with spinal cord injury," The Journal of Rehabilitation Research and Development, vol. 50, no. 2, pp. 203-214, 2013.

[27] P. Serra-Añó, L. L. Montesinos, J. Morales et al., "Heart rate variability in individuals with thoracic spinal cord injury," Spinal Cord, vol. 53, no. 1, pp. 59-63, 2015.

[28] S. M. Yu1, S. Y. Chen, and K. H. Lin, "The immediate effect of wheelchair Tai Chi on autonomic nervous modulation in subjects with chronic spinal cord injury: a pilot study," International Sport Med Journal, vol. 13, no. 3, pp. 96-108, 2012.

[29] S. J. Motivala, J. Sollers, J. Thayer, and M. R. Irwin, "Tai Chi Chih acutely decreases sympathetic nervous system Activity in older adults," The Journals of Gerontology Series A: Biological Sciences and Medical Sciences, vol. 61, no. 11, pp. 1177-1180, 2006.

[30] D. B. Irwin, C. C. Oyarce, and R. S. Plaza, "Effects of spinal cord injury in heart rate variability after acute and chronic exercise: a systematic review," Topics in Spinal Cord Injury Rehabilitation, vol. 24, no. 2, pp. 167-176, 2018.

[31] J. Sztajzel, "Heart rate variability: a non-invasive electrocardiographic method to measure the autonomic nervous system," Swiss Medical Weekly, vol. 34, pp. 514-522, 2004.

[32] F. Giagkoudaki, E. Dimitros, E. Kouidi, and A. Deligiannis, "Effects of exercise training on heart-rate-variability indices in individuals with down syndrome," Journal of Sport Rehabilitation, vol. 19, no. 2, pp. 173-183, 2010.

[33] M. T. La Rovere, J. T. Bigger Jr., F. I. Marcus et al., "Baroreflex sensitivity and heart-rate variability in prediction of total cardiac mortality after myocardial infarction. ATRAMI (autonomic tone and reflexes after myocardial infarction) investigators," Lancet, vol. 351, no. 9101, pp. 478-484, 1998.

[34] F. Lombardi and P. K. Stein, "Origin of heart rate variability and turbulence: an appraisal of autonomic modulation of cardiovascular function," Frontiers in Physiology, vol. 2, p. 95, 2011.

[35] S. Akselrod, D. Gordon, F. A. Ubel, D. Shannon, A. Berger, and R. Cohen, "Power spectrum analysis of heart rate fluctuation: a quantitative probe of beat-to-beat cardiovascular control," Science, vol. 213, no. 4504, p. 220, 1981.

[36] G.-X. Wei, Y.-F. Li, X.-L. Yue et al., "Tai Chi Chuan modulates heart rate variability during abdominal breathing in elderly adults," PsyCh Journal, vol. 5, no. 1, pp. 69-77, 2016.

[37] R. Perini and A. Veicsteinas, "Heart rate variability and autonomic activity at rest and during exercise in various physiological conditions," European Journal of Applied Physiology, vol. 90, no. 3-4, pp. 317-325, 2003.

[38] J. N. Myers, L. Hsu, D. Hadley, M. Y. Lee, and B. J. Kiratli, "Post-exercise heart rate recovery in individuals with spinal cord injury," Spinal Cord, vol. 48, no. 8, pp. 639-644, 2010.

[39] F. A. Cunha, A. W. Midgley, T. Gonçalves et al., "Parasympathetic reactivation after maximal CPET depends on exercise modality and resting vagal activity in healthy men," Springer Plus, vol. 4, p. 100, 2015.

[40] Y. Huang and X. Liu, "Improvement of balance control ability and flexibility in the elderly Tai Chi Chuan (TCC) practitioners: a systematic review and meta-analysis," Archives of Gerontology and Geriatrics, vol. 60, no. 2, pp. 233-238, 2015.

[41] D. L. Jardine, I. C. Melton, I. G. Crozier et al., "Decrease in cardiac output and muscle sympathetic activity during vasovagal syncope," American Journal of Physiology-Heart and Circulatory Physiology, vol. 282, no. 5, pp. H1804-H1809, 2002.

[42] D. S. Goldstein, O. Bentho, M.-Y. Park, and Y. Sharabi, "Lowfrequency power of heart rate variability is not a measure of cardiac sympathetic tone but may be a measure of modulation of cardiac autonomic outflows by baroreflexes," Experimental Physiology, vol. 96, no. 12, pp. 1255-1261, 2011.

[43] A. R. Kiselev, E. I. Borovkova, V. A. Shvartz et al., "Lowfrequency variability in photoplethysmographic waveform and heart rate during on-pump cardiac surgery with or without cardioplegia," Scientific Reports, vol. 10, no. 1, p. 2118, 2020. 